\title{
Structural modifications of timothy lignin by in vitro rumen fermentation
}

\author{
T Kondo, T Ohshita, T Kyuma \\ Tohoku National Agricultural Experiment Station, Shimokuriyagawa, Morioka 020-01, Japan
}

Lignin in forages is accepted as one of the most important chemical components due to its key role in ruminal degradation of forage fibers. The nutritional effect of forage lignin is closely related to its structure (Besle et al, 1994, J Sci Food Agric, 64, 171-190) and thus probably affected by changes that forage lignin undergoes in the digestive tract. Rumen fermentation has been reported to cause decomposition of a lignin model dimmer into monoaromatic compounds (Chen et al, 1985, Appl Environ Microbiol, 50, 1451-1456) and demethylation of the syringyl units of grass lignin (Mossoni et al, 1994, J Sci Food Agric, 64, 379387 ), but no further evidence is presented showing structural degradation of forage lignin by rumen fermentation. The objective of the present study was to investigate structural modifications of forage grass lignin occurring in the rumen.

A lignin fraction (FHL) was isolated with $90 \%$ dioxane from the ball-milled and in vitro rumen fermented residue of timothy hay harvested at the seed setting stage. The lignin fraction was compared with $90 \%$ dioxane-soluble lignins isolated from the ball-milled and cellulasetreated residue of the timothy hay $(E H L)$ and from the faeces of a heifer fed on the timothy hay (FSL).

FHL consisted of more lignin components, less carbohydrate and less bound phenolic acids than EHL and had a compositional resemblance to FSL (Table). Alkaline nitrobenzene oxidation and IR spectra showed all the lignins to be guaiacyl-syringyl lignins. In gel permeation chromatograms, FHL and FSL were distributed in lower molecular size regions than EHL.

Lignin modifications occurring in the rumen have been often reported and the observed major modification is solubilisation of lignin. Release of lignin due to solubilisation was considered as a consequence of degradation of surrounding polysaccharides in the rumen (Conchie et al, 1988, Carbohydr Res, 177, 127-151). The release of $E H L$ from the ball-milled timothy is also based on the enzymatic degradation of associated polysaccharides. FHL, produced by the in vitro rumen fermentation of the ball-milled timothy, differed in chemical and physical properties from EHL, suggesting removal or degradation of the phenolic acids bound to timothy lignin and depolymerisation of timothy lignin by rumen fermentation.

\begin{tabular}{lc} 
Chemical components & EHL \\
\hline Klason lignin & 716 \\
Total carbohydrate & $\mathbf{1 8 9}$ \\
Esterified p-coumaric acid & 15.6 \\
Esterified ferulic acid & 2.1 \\
\hline
\end{tabular}

$\mathrm{FHL}$ ( $g / k g$ lignin fraction)

$\begin{array}{cc}887 & 890 \\ 50 & 37 \\ 3.7 & 3.5 \\ 0.4 & 0.5\end{array}$

\section{FSL}

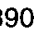

3.5 